\title{
Graphical Analysis of q-Rung Orthopair Fuzzy Information with Application
}

\author{
Hussain AlSalman $\mathbb{D D}^{1}$ and Bader Fahad Alkhamees $\mathbb{D}^{2}$ \\ ${ }^{1}$ Department of Computer Science, College of Computer and Information Sciences, King Saud University, \\ Riyadh 11543, Saudi Arabia \\ ${ }^{2}$ Department of Information Systems, College of Computer and Information Sciences, King Saud University, \\ Riyadh 11543, Saudi Arabia \\ Correspondence should be addressed to Hussain AlSalman; halsalman@ksu.edu.sa
}

Received 2 November 2021; Accepted 29 December 2021; Published 27 February 2022

Academic Editor: Naeem Jan

Copyright $\odot 2022$ Hussain AlSalman and Bader Fahad Alkhamees. This is an open access article distributed under the Creative Commons Attribution License, which permits unrestricted use, distribution, and reproduction in any medium, provided the original work is properly cited.

\begin{abstract}
The q-rung orthopair fuzzy graph (q-ROFG) is an expansion of the intuitionistic fuzzy graph (IFG) and Pythagorean fuzzy graph (PFG); q-rung orthopair fuzzy model is an influential model for describing vagueness and uncertainty as a comparison to an intuitionistic fuzzy model and Pythagorean fuzzy model. The research aims to illustrate the notion of the graph of q-rung orthopair fuzzy sets (q-ROFSs). Furthermore, in this article, we examine the ideas of domination theory (DT) and double domination theory (DDT) in q-ROFGs. Additionally, the structure of q-ROFG is developed and its associated concept is presented through the assistance of instructive instances. Furthermore, the DT of q-ROFGs is established, as are cardinality, power, and completeness on dominance in a q-ROFG and bipartite q-ROFG, and double domination set (DDS), as well as some results, is investigated in the concept of q-ROFGs. A political campaign is simulated using the proposed structure as an application, and the impact of double dominance (DD) on political campaigns is investigated. Finally, a comparison is given between the proposed study and actual studies, as well as the advantages of working in the q-ROFG scenario.
\end{abstract}

\section{Introduction}

In 1965, Zadeh [1] initiated the notion of the fuzzy set (FS). The FS theory has been shown to be a useful tool for defining scenarios with uncertain or imprecise data. FS is an expansion of the crisp set. The intuitionistic fuzzy set (IFS) was first proposed by Atanassov [2]; here we can define the level of membership (LM) and the level of nonmembership (LNM) of an object. The IFS is an extension of FS which can be used in instances where FS is unsuitable. Yager [3] proposed the notion of Pythagorean fuzzy sets (PFSs) in 2013. PFS is an expansion of FS and IFS. As a consequence, it is better able to express and handle fuzzy data in practice and scientific study. The notion of q-ROFSs was also established by Yager [4], q-ROFS is an extension of IFS and PFS, and the major feature of q-ROFS is that the LM and LNM have a broader uncertain space. A q-ROFS is additionally more useful and efficient than IFS or a PFS to describe uncertainty in different decision-making issues.

Kaufman [5] and Rosenfeld [6] proposed the notion of fuzzy graphs (FGs) as the generalization of crisp graphs. The vertices and edges of an FG are fuzzy numbers; therefore, a variety of investigators have applied the idea of FGs to realworld problems; for example, Sameena and Sunitha [7] utilized FGs in fuzzy neural networks, Sunitha and Mathew [8] investigated FG theory, and Yeh and Bang [9] used FGs in clustering analysis and cognitive and decision-making processes. IFG is expanded by Parvathi and Karunambigai [10], which is an extension of the FG. As previously stated, IFS is more beneficial than FS, and the same is true for IFGs and FGs. Talebi et al. [11] worked on new idea of IFG with application. Rangasamy et al. [12] worked out in a network the intuitionistic fuzzy shortest hyperpath. The IFSs and IFGs were extensively explored and presented by Akram and 
Al-Shehrie [13]. Bozhenyuk et al. [14] worked on the dominance set in IFG.

Graph theory has been applied to a variety of fields. The DT and its uses are very prominent in graph theory. Sigaretta initiated the concept of total dominance on Certain Graphical Functions [15]. Different forms of dominance were developed by Karunambigai et al. [16] in IFG. DT is extremely useful in FGs and IFGs and has several applications. Somasundaram and Somasundaram [17] initiated the concept of DT of FGs, while Parvathi and Thamizhendhi [18] introduced the domination in IFGs. Additionally, [19, 20] addressed some features of strong domination in FGs. Borzooei and Rashmanlou [21] compiled and described the ideas of FG domination and their applications. Manjusha and Sunitha [22] presented some concepts in FGs; Zhang et al. [23] discussed how dynamic dominance develops in fuzzy causal systems. Shubatah [24] explored the notions of dominance and total domination in FGs. Jan et al. [25] investigated interval-valued PFGs decision-making and shortest path issues. Pachamuthu and Praveenkumar [26] introduced the idea of dominance in IFGs of second type. The graphs for $\mathrm{n}^{\text {th }}$ type IFSs and their applications were presented by Davvaz et al. [27].

The q-ROFG theory, which is based on q-ROFSs, was introduced by Habib and Farooq [28]; q-ROFGs are a generalization of IFGs and PFGs. FG simply addresses the level of membership, whereas IFGs, PFGs, and q-ROFGs describe both levels of membership and the level of nonmemberships. Yin et al. [29] worked on product operations on q-ROFGs. Zeng et al. [30] expanded the concepts of q-rung orthopair fuzzy weight induced logarithm. Wan et al. [31] worked on the weight average LINMAP group decisionmaking based on q-rung orthopair fuzzy triangular. Peng et al. [32] worked on the q-rung orthopair fuzzy decisionmaking system which was developed for implementing mobile edge caching method preferences. Peng and Luo [33] introduced a study that collects a total of 80 publications related to q-ROFS in Web of Science for in-depth analysis. Roughly important results regarding the country, annual trends level, journal level, institutional level, and research landscape and highly cited papers are illustrated and generated. They summarized eighteen research challenges or future directions for the q-ROFS theory. Therefore, the motivation is given by the need to examine the ideas of domination theory (DT) and double domination theory (DDT) in q-ROFGs.

This article is arranged as follows. In Section 1, we give a quick overview of some basic definitions. In Section 2, we look through several essential terminologies and ideas associated with IFGs theory and q-ROFGs. In Section 3, we extend the notion of q-ROFGs, q-rung orthopair fuzzy subgraph (q-ROFSG), and the component of edge relationship, bridge, and cut-vertices. In Section 4, we clearly illustrate the notion of dominance in a q-rung orthopair fuzzy environment. Section 5 presents the idea of q-ROFG double dominance and related terminology. In part 6 , we discuss an innovative use of double domination in a candidate's political campaign in a constituency. In part 7, we conduct a comparative analysis of the proposed methodologies with previous work dominance. Eventually, in part 8, we provide a summary of the study.

\section{Preliminaries}

In this portion, we will go over various essential ideas associated with the theory of FGs, IFGs, intuitionistic fuzzy subgraph (IFSG), and q-ROFSs. In this part, we will also examine the DT of IFSs.

Definition 1 (see [5]). A pair $F=(\check{C}, \check{D})$ is said to be FG if

(i) $\check{C}=\left\{c_{1}, c_{2}, c_{3}, \ldots, c_{i}\right\}$ is the set of vertices, and $\xi_{\check{C}}: \stackrel{C}{\longrightarrow}[0,1]$ represents the LM of $c_{i}$ in $\check{C}$ such that $0 \leq \xi_{\check{C}}\left(c_{i}\right) \leq 1$.

(ii) $\check{\mathrm{D}}=\left\{\mathrm{d}_{1}, \mathrm{~d}_{2}, \mathrm{~d}_{3}, \ldots, \mathrm{d}_{\mathrm{i}}\right\}$ is the set of edges, and $\check{\mathrm{D}} \subseteq \check{C} \times \check{\mathrm{C}}$. The mapping $\xi_{\check{\mathrm{D}}}: \check{\mathrm{C}} \times \check{\mathrm{C}} \longrightarrow[0,1]$ represents the LM of $d_{i}$, where $\xi_{\mathrm{D}}\left(c_{i}, c_{j}\right) \leq$ $\min \left\{\xi_{\check{C}}\left(c_{i}\right), \xi_{\check{C}}\left(c_{j}\right)\right\}$ for $i, j \in \mathbb{N}$. The condition of $0 \leq \xi_{\mathrm{D}}\left(c_{i}, c_{j}\right) \leq 1$ is satisfied.

Definition 2 (see [10]). A pair $F=(\check{C}, \check{D})$ is said to be IFG if

(i) $\check{C}=\left\{c_{1}, c_{2}, c_{3}, c_{4}, \ldots, c_{i}\right\}$ is the set of vertices, and $\xi_{\check{C}}: \stackrel{\mathrm{C}}{\longrightarrow}[0,1]$ and $\psi_{\check{C}}: \check{\mathrm{C}} \longrightarrow[0,1]$ show the LM and LNM of $c_{i}$ in $\check{C}$, respectively, such that

$$
0 \leq \xi_{\check{C}}\left(c_{i}\right)+\psi_{\check{C}}\left(c_{i}\right) \leq 1 .
$$

(ii) $\check{\mathrm{D}}=\left\{\mathrm{d}_{1}, \mathrm{~d}_{2}, \mathrm{~d}_{3}, \ldots, \mathrm{d}_{\mathrm{i}}\right\}$ is the set of edges, and $\check{\mathrm{D}} \subseteq \check{C} \times \check{C}$. The mappings $\xi_{\check{D}}: \check{\mathrm{C}} \times \check{\mathrm{C}} \longrightarrow[0,1]$ and $\psi_{\check{\mathrm{D}}}: \check{\mathrm{C}} \times \check{\mathrm{C}} \longrightarrow[0,1]$ are known as the LM and the LNM of $d_{i}$, respectively, where $\xi_{\check{D}}\left(c_{i}, c_{j}\right) \leq \min$ $\left\{\xi_{\check{C}}\left(c_{i}\right), \xi_{\check{C}}\left(c_{j}\right)\right\} \quad$ and $\psi_{\check{D}}\left(c_{i}, c_{j}\right) \leq \max \left\{\psi_{\check{C}}\left(c_{i}\right)\right.$, $\left.\psi_{\check{C}}\left(c_{j}\right)\right\}$ for $i, j \in \mathbb{N}$.

$$
0 \leq \xi_{\check{D}}\left(c_{i}, c_{j}\right)+\psi_{\check{D}}\left(c_{i}, c_{j}\right) \leq 1
$$

Example 1. Let $F=(\check{C}, \check{D})$ be an IFG in Figure 1 , let $\check{C}=$ $\left\{c_{1}, c_{2}, c_{3}, c_{4}\right\}$ be the collection of vertices, and let $\stackrel{\mathrm{D}}{ }=\left\{\mathrm{c}_{1} \mathrm{c}_{2}, \mathrm{c}_{3} \mathrm{c}_{4}, \mathrm{c}_{2} \mathrm{c}_{4}, \mathrm{c}_{1} \mathrm{c}_{3}\right\}$ be the collection of edges.

Definition 3 (see [10]). A pair $F=(\check{C}$, $\check{D})$ is called IFG; then an IFSG is of the form $F^{\prime}=\left(\check{C}^{\prime}, \check{\mathrm{D}}^{\prime}\right)$ if $\check{C}^{\prime} \subseteq \check{C}$ and $\check{\mathrm{D}}^{\prime} \subseteq \check{\mathrm{D}}$, where $\xi_{\check{\mathrm{C}}}\left(c_{i}\right) \leq \xi_{\check{\mathrm{C}}}\left(c_{i}\right), \quad \xi_{\check{\mathrm{D}}^{\prime}}\left(d_{i}\right) \leq \xi_{\check{\mathrm{D}}}\left(d_{i}\right), \quad \psi_{\check{\mathrm{C}}^{\prime}}\left(c_{i}\right) \geq \psi_{\check{\mathrm{C}}}\left(c_{i}\right)$, and $\psi_{\check{\mathrm{D}}^{\prime}}\left(d_{i}\right) \geq \psi_{\check{\mathrm{D}}}\left(d_{i}\right)$, for $i \in \mathbb{N}$.

Definition 4 (see [18]). Let $F=(\check{C}, \check{D})$ be an IFG; then, for any $c_{1}, c_{2} \in \check{\mathrm{D}}, c_{1}$ is said to dominate $c_{2}$ in $F$ if $\xi_{\check{\mathrm{D}}}\left(c_{1}, c_{2}\right)=$ $\min \left\{\xi_{\check{C}}\left(c_{1}\right), \xi_{\check{C}}\left(c_{2}\right)\right\}$ and $\psi_{\check{\mathrm{D}}}\left(c_{1}, c_{2}\right)=\max \left\{\psi_{\check{\mathrm{C}}}\left(c_{1}\right), \psi_{\check{\mathrm{C}}}\left(c_{2}\right)\right\}$. For $\check{C}^{\prime} \subseteq \check{C}$, if, $\forall c^{\prime} \notin \check{C}^{\prime}, \exists c \in \check{C} \ni c$ dominates $c^{\prime}$, then $\check{C}$ is called the DS in $F$. The dominant number (DN) of an IFG $F$ is the fuzzy cardinality of DS with the smallest fuzzy cardinality in $F$. The DN of $F$ is symbolized as $\varphi(F)$. 


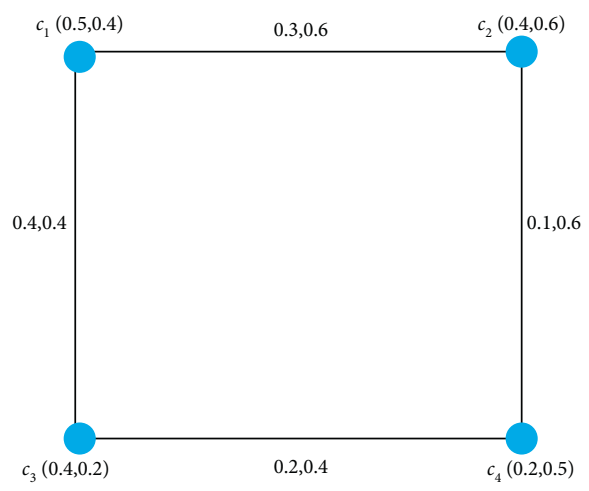

FIGURE 1: IFG.

\section{Remark 1}

(i) For any $c_{1}, c_{2} \in \check{\mathrm{C}}$, if $c_{1}$ dominates $c_{2}$, then $c_{2}$ dominates $c_{1}$ in $F$; it displays the symmetry of domination

(ii) If $\xi_{\check{D}}\left(c_{1}, c_{2}\right)=\min \left\{\xi_{\check{C}}\left(c_{1}\right), \xi_{\check{C}}\left(c_{2}\right)\right\}$ and $\psi_{\check{D}}\left(c_{1}, c_{2}\right)=$ $\max \left\{\psi_{\check{C}}\left(c_{1}\right), \psi_{\check{C}}\left(c_{2}\right)\right\}$, for all $c_{1}, c_{2} \in \check{C}$, then clearly the only DS in $F$ is $\check{C}$

Definition 5 (see [18]). The strong edge of the edge $\left(c_{1}, c_{2}\right)$ in an IFG is of the form $\xi_{\check{D}}\left(c_{1}, c_{2}\right) \geq \xi_{\mathrm{D}}^{\infty}\left(c_{1}, c_{2}\right)$, where $\psi_{\check{\mathrm{D}}}\left(c_{1}, c_{2}\right) \geq \psi_{\check{\mathrm{D}}}^{\infty}\left(c_{1}, c_{2}\right)$.

Definition 6 (see [18]). If there is no proper dominating subset, the DS $S$ of an IFG is known as minimum DS.

Definition 7 (see [18]). If there is a strong independent edge between a couple of vertices in an IFG $F$, they are considered independent.

Definition 8 (see [4]). Let $\check{C}$ be a q-ROFS. $\check{C}$ in $E$ is defined as $\check{\mathrm{C}}=\{\mathrm{e}, \xi(\mathrm{e}), \psi(\mathrm{e}) \mid \mathrm{e} \in \mathrm{E}\}$, where $\xi(e): \longrightarrow[0,1]$ shows the LM and $\psi(e): \longrightarrow[0,1]$ denotes the LNM and the following condition is satisfied:

$$
0 \leq \xi_{\check{C}}^{n}(e)+\psi_{\check{C}}^{n}(e) \leq 1 \forall e \in E .
$$

The double $(\xi, \psi)$ represents the q-rung orthopair fuzzy number (q-ROFN).

\section{3. q-Rung Orthopair Fuzzy Graphs}

In this part, we develop the idea of q-ROFG, q-rung orthopair fuzzy subgraph (q-ROFSG), and the relationship between edges, bridge, and cut-vertices. With the help of examples, these ideas are demonstrated.

Definition 9 (see [28]). A pair $F=(\check{C}, \check{D})$ is said to be q-ROFG if we have the following:

(i) $\check{C}=\left\{c_{1}, c_{2}, c_{3}, \ldots, c_{i}\right\}$ is the collection of vertices, and $\xi_{\check{C}}: \check{C} \longrightarrow[0,1]$ and $\psi_{\check{C}}: \check{C} \longrightarrow[0,1]$ show the LM and LNM of $c_{i}$ in $\check{C}$ such that

$$
0 \leq \xi_{\check{C}}^{n}\left(c_{i}\right)+\psi_{\check{C}}^{n}\left(c_{i}\right) \leq 1 .
$$

(ii) $\stackrel{\mathrm{D}}{=}=\left\{\mathrm{d}_{1}, \mathrm{~d}_{2}, \mathrm{~d}_{3}, \ldots, \mathrm{d}_{\mathrm{i}}\right\}$ is the set of edges, and $\check{\mathrm{D}} \subseteq \mathrm{C} \times \check{C}$. The mappings $\xi_{\check{D}}: \check{C} \times \check{C} \longrightarrow[0,1]$ and $\psi_{\check{\mathrm{D}}}: \check{\mathrm{C}} \times \check{\mathrm{C}} \longrightarrow[0,1]$ indicate the LM and LNM of $d_{i}$, correspondingly, where $\xi_{\check{D}}\left(c_{i}, c_{j}\right) \leq \min$ $\left\{\xi_{\check{C}}\left(c_{i}\right), \xi_{\check{C}}\left(c_{j}\right)\right\} \quad$ and $\quad \psi_{\check{\mathrm{D}}}\left(c_{i}, c_{j}\right) \leq \max \left\{\psi_{\check{\mathrm{C}}}\left(c_{i}\right)\right.$, $\left.\psi_{\check{C}}\left(c_{j}\right)\right\}$ for $i, j \in \mathbb{N}$.

$$
0 \leq \xi_{\overline{\mathrm{D}}}^{n}\left(c_{i}, c_{j}\right)+\psi_{\mathrm{D}}^{n}\left(c_{i}, c_{j}\right) \leq 1
$$

Example 2. In Figure 2, $F=(\check{\mathrm{C}}, \check{\mathrm{D}})$ is a q-ROFG, $\check{C}=\left\{c_{1}, c_{2}, c_{3}, c_{4}\right\} \quad$ is a collection of vertices, and $\check{D}=\left\{c_{1} c_{2}, c_{2} c_{3}, c_{3} c_{4}, c_{4} c_{1}\right\}$ is a collection of edges.

Figure 2 is simply q-ROFG for $n=4$.

Remark 2. IFGs and PFGs are $\mathrm{q}-\mathrm{ROFG}$ but the inverse is not true.

Definition 10. Let $F=(\check{C}, \check{D})$ be a q-ROFG; then q-ROFSG is of the form $F^{\prime}=\left(\check{C}^{\prime}, \check{D}^{\prime}\right)$ such that $\check{C}^{\prime} \subseteq \check{C}$ and $\check{D}^{\prime} \subseteq \check{D} . \xi_{\check{C}}$ and $\xi_{\check{D}^{\prime}}$ represent the LM and $\psi_{\check{C}^{\prime}}, \psi_{\check{D}^{\prime}}$ represent the LNM, such that $\xi_{\check{C}^{\prime}}^{n}\left(c_{i}\right) \leq \xi_{\check{\mathrm{C}}}^{n}\left(c_{i}\right), \quad \xi_{\check{\mathrm{D}}^{\prime}}^{n}\left(d_{i}\right) \leq \xi_{\check{D}^{\prime}}^{n}\left(d_{i S}\right), \quad \psi_{\check{C}^{\prime}}^{n}\left(c_{i}\right) \geq$ $\psi_{\check{\mathrm{C}}}^{n}\left(c_{i}\right)$, and $\psi_{\check{\mathrm{D}}^{\prime}}^{n}\left(d_{i}\right) \geq \psi_{\check{\mathrm{D}}}^{n}\left(d_{i}\right)$, for $i \in \mathbb{N}$.

Definition 11. $\left(c_{i}, c_{j}\right)$ is an edge in a q-ROFG $F=(\check{C}, \check{D})$. If removing this edge reduces the power of connectivity between pairs of vertices in $F$, it is called a bridge.

Example 3. Let $F=(\check{C}, \check{D})$ be a q-ROFG in Figure 3, and $\check{C}=\left\{c_{1}, c_{2}, c_{3}, c_{4}\right\}$ is a set of vertices and $\check{D}=\left\{c_{1} c_{2}, c_{2} c_{3}\right.$, $\left.c_{3} c_{1}, c_{3} c_{4}\right\}$ is a set of edges.

In Figure $3,\left(c_{3}, c_{4}\right)$ is a bridge.

Definition 12. The combination of two edge relationships

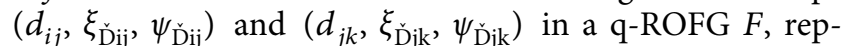
resented by $d_{i j}{ }^{o} d_{j k}$, is of the following form: $\left(d_{i k}, \xi_{\text {Dik }}, \psi_{\text {Dik }}\right)$; here, $\xi_{\text {Dik }}=\max \left\{\min _{j}\left[\xi_{\text {Dij }}, \xi_{\text {D }}\right.\right.$ jk $\left.]\right\}$ and $\psi_{\text {Dik }}=\operatorname{mini}\left\{\max _{j}\left[\psi_{\text {Dij }}, \psi_{\text {D } j \mathrm{k}}\right]\right\}, \forall c_{i}, c_{k} \in \check{C}$.

Definition 13. If $d_{i j}$ is an edge connection of q-ROFG $F$, then the power of $d_{i j}$ is defined as

$$
\begin{aligned}
& 1_{i j}=d_{i j}=\left(d_{i j}, \xi_{\check{D} \mathrm{ij}}, \psi_{\mathrm{D} i \mathrm{ij}}\right), \\
& { }_{d_{i j}}^{2}=d_{i j}{ }^{\circ} \mathrm{d}_{\mathrm{ij}}=\left(d_{i j}, \xi_{\mathrm{D} \mathrm{ij}}^{2}, \psi_{\mathrm{D} \mathrm{ij}}^{2}\right) \text {, } \\
& { }_{d_{i j}}^{3}=d_{i j}{ }^{\circ} \mathrm{d}_{\mathrm{ij}}{ }^{\circ} \mathrm{d}_{\mathrm{ij}}=\left(d_{i j}, \xi_{\mathrm{D} \mathrm{ij}}^{3}, \psi_{\mathrm{D} \mathrm{ij}}^{3}\right) \text {, }
\end{aligned}
$$

and so on.

$$
{ }_{d_{i j}}^{\infty}=\left(d_{i j}, \xi_{\mathrm{D} \mathrm{ij}}^{\infty}, \psi_{\mathrm{D} \mathrm{ij}}^{\infty}\right)
$$

Here, $\xi_{\text {Dij }}^{\infty}=\max _{y=1,2, \ldots n}\left\{\xi_{\text {Dij }}^{y}\right\}$ and $\psi_{\text {Dij }}^{\infty}=\min _{y=1,2, \ldots n}$ $\left\{\psi_{\mathrm{D} i \mathrm{ij}}^{y}\right\}$ are the LM strength and the LNM strength of connecting between two vertices of $c_{i}$ and $c_{j}$. 


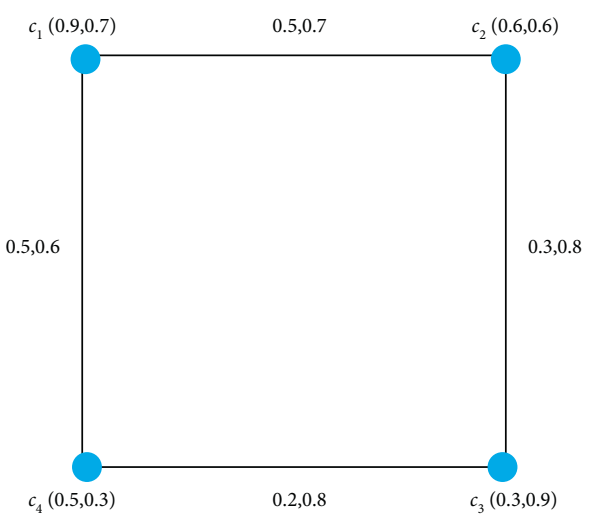

Figure 2: The q-ROFG.

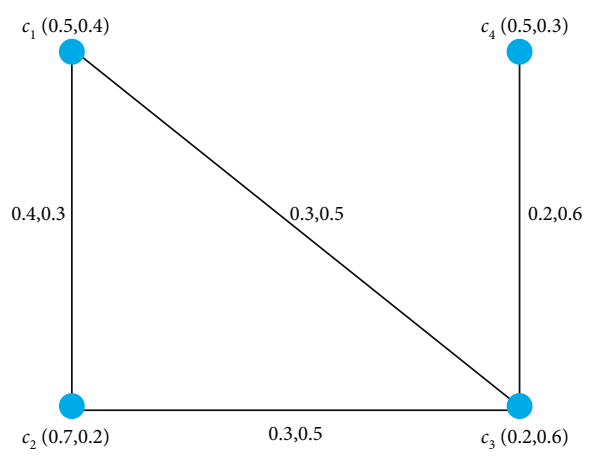

Figure 3: The q-ROFG for bridge.

We have

${\stackrel{0}{\mathrm{~d}_{i j}}}_{i}=\left\{0\right.$, if $c_{i} \neq c_{j}$ and $\left(c_{i}, \xi_{\mathrm{C} \mathrm{i}}, \psi_{\mathrm{C} \mathrm{i}}\right)$ if $c_{i}$ is equal to $\left.c_{j}\right\}$.

Theorem 1. If $d_{i j}=\left(c_{i}, c_{j}\right)$ is an edge in $F$, then, for any two vertices in $q$-ROFG $F=(\check{C}, \check{D})$, the following statements are equipped:

(i) $d_{i j}$ is a bridge

(ii) $\xi_{D i j}^{* \infty}<\xi_{\check{D} i j}$, and $\psi_{D i j}^{* \infty}>\psi_{\bar{D} i j}$

(iii) $d_{i j}$, there is no cycle's edge

Proof: We will do it as follows: $(i i) \Rightarrow(i) \Rightarrow(i i i) \Rightarrow(i i)$.

$(i i) \Rightarrow(i)$. Let $\xi_{\text {Dij }}^{* \infty}<\xi_{\text {Dij }}$ and $\psi_{\text {Dij }}^{* \infty}>\psi_{\text {Dij }}$. To demonstrate that (i) is accurate, we suppose that the opposite is true. After that,

$$
\xi_{\text {Dij }}^{* \infty}=\xi_{\text {D้ij }}^{\infty} \geq \xi_{\text {Ďij }} \text {, and } \psi_{\text {Dij }}^{* \infty}=\psi_{\text {Dij }}^{\infty} \leq \psi_{\text {Ďij }}
$$

$\Rightarrow \xi_{\mathrm{Dij}}^{* \infty} \geq \xi_{\text {Ďij }}$, and $\psi_{\mathrm{D} \mathrm{ij}}^{* \infty} \leq \psi_{\mathrm{D} i \mathrm{ij}}, \quad$ resulting in a contradiction, so (i) is true.

$(i) \Rightarrow(i i i)$. Now, we let $\mathrm{d}_{i j}$ be a cycle's edge; then, containing edge $\mathrm{d}_{i j}$ to any path can be converted onto a path that does not contain $\left(c_{i}, c_{j}\right)$. A cycle is a route from $c_{i}$ to $c_{j} \Rightarrow \mathrm{d}_{i j}$ which is not a bridge, since it is a contradiction. Thus, $\mathrm{d}_{i j}$ is not a cycle's edge. $(i i i) \Rightarrow(i i)$. The conclusion is simple.

Definition 14. $c_{i}$ is a vertex in a q-ROFG $F=(\check{C}, \check{\mathrm{D}})$; if removing this vertex reduces the power of connectivity between some vertices in $F$, it is called a cut-vertex.

Example 4. Suppose that $F=(\check{C}, \check{D})$ is a q-ROFG in Figure 4 , and $\check{C}=\left\{c_{1}, c_{2}, c_{3}, c_{4}, c_{5}, \ldots, c_{i}\right\}$ is a set of vertices and $\check{D}=\left\{c_{1} c_{2}, c_{2} c_{4}, c_{4} c_{5}, c_{4} c_{3}, c_{3} c_{1}\right\}$ is a set of edges.

In Figure $4, n=2$, and $c_{3}$ is a cut-vertex .

\section{Domination in q-Rung Orthopair Fuzzy Graph}

In this part, for q-ROFGs, we introduced the ideas of cardinality, power, and completeness. Additionally, we define the domination in $\mathrm{q}-\mathrm{ROFG}$ and prove it with some results. First, we define vertex cardinality (VC) and edge cardinality (EC).

Definition 15. Let $F=(\check{C}, \check{D})$ be a q-ROFG. The VC of $\check{C}$ is defined and represented by

$$
|\check{C}|=\sum_{\mathrm{c}_{\mathrm{i}} \in \check{\mathrm{C}}} \frac{1+\xi_{\check{C}}\left(\mathrm{c}_{\mathrm{i}}\right)-\psi_{\check{\mathrm{C}}}\left(\mathrm{c}_{\mathrm{i}}\right)}{2}, \forall \mathrm{c}_{\mathrm{i}} \in \check{\mathrm{C}} \text {, }
$$

where $\left(\xi_{\check{C}}\left(c_{i}\right), \psi_{\mathrm{C}}\left(c_{i}\right)\right)$ shows the level of membership and the level of nonmembership.

Definition 16. Let $F=(\check{C}, \check{D})$ be a q-ROFG. Then, the EC of $\mathrm{D}$ is represented and defined by

$$
|\check{D}|=\sum_{c_{i}, c_{j} \in D^{\prime}} \frac{1+\xi_{\check{D}}\left(c_{i}, c_{j}\right)-\psi_{\check{D}}\left(c_{i}, c_{j}\right)}{2}, \forall c_{i}, c_{j} \in \check{D}
$$

where $\xi_{\check{D}}\left(c_{i}, c_{j}\right), \psi_{\check{\mathrm{D}}}\left(c_{i}, c_{j}\right)$ show the level of membership and the level of nonmembership.

Definition 17. The cardinality of a q-ROFG $F=(\check{C}, \check{D})$ is defined and represented by

$|F|=\left|\sum_{c_{i} \in \check{\mathrm{C}}} \frac{1+\xi_{\check{\mathrm{C}}}\left(c_{i}\right)-\psi_{\check{\mathrm{C}}}\left(c_{i}\right)}{2}+\sum_{c_{i}, c_{j} \in \check{\mathrm{D}}} \frac{1+\xi_{\check{\mathrm{D}}}\left(c_{i}, c_{j}\right)-\psi_{\check{\mathrm{D}}}\left(c_{i}, c_{j}\right)}{2}\right|$.

Remark 3. In a $\mathrm{q}-\mathrm{ROFG}$, the order of $\mathrm{q}-\mathrm{ROFG}$ means the collection of vertices, and it is indicated by $\mathcal{O}(F)$. The size of a q-ROFG is defined as the set of edges in the q-ROFG $F$, and it is indicated by $S(F)$.

Definition 18. For $F=(\check{C}, \stackrel{\mathrm{D}}{)})$ is a $\mathrm{q}-\mathrm{ROFG}$, the degree of vertex $c_{i}$ is defined as the sum of the weight of SE occurrence to $c_{i}$, and it is represented by $k_{F}\left(c_{i}\right)$. The least degree of $F$ is $\delta(F)=\min \left\{k_{F}\left(c_{i}\right): c_{i} \in \check{C}\right\}$, and the maximum degree of $F$ is $\Delta(F)=\max \left\{k_{F}\left(c_{i}\right): c_{i} \in \check{C}\right\}$. 


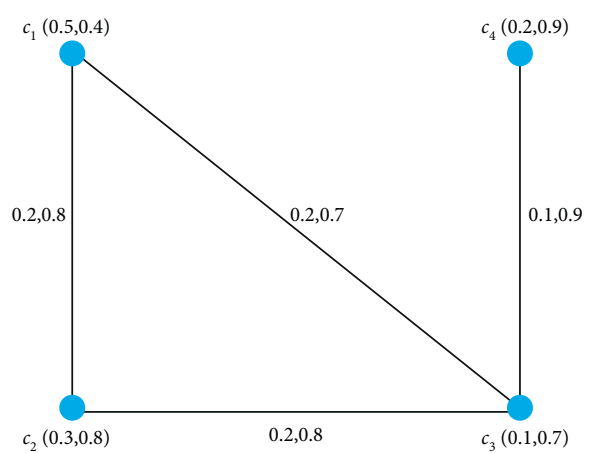

Figure 4: The q-ROFG for cut-vertex.

Definition 19. In a q-ROFG, a couple of vertices $c_{i}$ and $c_{j}$ are considered to be neighbors, if one or more of the following attributes is accurate:
(i) $\xi_{\check{D}}\left(c_{i}, c_{j}\right)>0, \psi_{\check{D}}\left(c_{i}, c_{j}\right)>0$
(ii) $\xi_{\check{D}}\left(c_{i}, c_{j}\right)=0, \psi_{\check{D}}\left(c_{i}, c_{j}\right)>0$
(iii) $\xi_{\check{D}}\left(c_{i}, c_{j}\right)>0, \psi_{\check{D}}\left(c_{i}, c_{j}\right)=0, c_{i}, c_{j} \in \check{C}$

Definition 20. In a q-ROFG, a path is a collection of various vertices such that $c_{1}, c_{2}, \ldots, c_{n}$. Then, one of the following requirements must be completed:

(i) $\xi_{\check{D}}\left(c_{i}, c_{j}\right)>0, \psi_{\check{D}}\left(c_{i}, c_{j}\right)>0$

(ii) $\xi_{\check{D}}\left(c_{i}, c_{j}\right)=0, \psi_{\check{D}}\left(c_{i}, c_{j}\right)>0$

(iii) $\xi_{\check{D}}\left(c_{i}, c_{j}\right)>0, \psi_{\check{D}}\left(c_{i}, c_{j}\right)=0 . c_{i}, c_{j} \in \check{C}$, for $i, j \in \mathbb{N}$

The length of a path is $\mathrm{P}=c_{1}, c_{2}, \ldots, c_{n+1}(n>0)$.

Remark 4. If a path connects a couple of vertices, they are said to be connected.

Definition 21. If $c_{i}$ and $c_{j}$ are a couple of vertices in q-ROFG $F$ associated using the path, then the path's strength is defined as $\left(\min _{i, j} \xi_{\check{D}_{\mathrm{Dij}}}, \max _{i, j} \psi_{\text {Dij }}\right)$, where $\min \xi_{\mathrm{D} \text { ij }}$ is the $\xi$-strength of the weakest edge and $\max _{i, j}^{i, j} \psi_{\mathrm{D}_{i j}}$ is the $\psi$-strength of the strongest edge.

Definition 22. If $c_{i}, c_{j} \in \check{\mathrm{C}} \subseteq \mathrm{F}$, then $\xi$-strength of connection between $c_{i}$ and $c_{j}$ is $\xi_{\overline{\mathrm{D}}}^{\infty}\left(c_{i}, c_{j}\right)=\sup \left\{\xi_{\mathrm{D}}^{x}\left(c_{i}, c_{j}\right)\right.$ : $x=1,2, . ., n\}$, and $\psi$-strength of connectivity between $c_{i}$ and $c_{j}$ is $\psi_{\grave{D}}^{\infty}\left(c_{i}, c_{j}\right)=\inf \left\{\psi_{\grave{D}}^{x}\left(c_{i}, c_{j}\right): x=1,2, . ., n\right\} ;$ if $c$ and $\hbar$ are associated by ways of the path of length $x$; then, $\xi_{\bar{D}}^{x}(c, \hbar)$ is defined as

$$
\begin{gathered}
\sup \left\{\xi_{\check{\mathrm{D}}}\left(c, \hbar_{1}\right) \min \xi_{\check{\mathrm{D}}}\left(\hbar_{1}, \hbar_{2}\right) \min \xi_{\check{\mathrm{D}}}\left(\hbar_{2}, \hbar_{3}\right) \ldots \min \xi_{\check{\mathrm{D}}}\right. \\
\left(\hbar_{x-1}, \hbar\right): c, \hbar, \hbar_{2}, \ldots, \hbar_{x-1}, \hbar \in \check{\mathrm{C}}
\end{gathered}
$$

$\psi_{\check{D}}^{x}(c, \hbar)$ is defined as

$$
\begin{aligned}
& \inf \left\{\psi_{\check{D}}\left(c, \hbar_{1}\right) \max \psi_{\check{D}}\left(\hbar_{1}, \hbar_{2}\right) \max \psi_{\check{D}}\left(\hbar_{2}, \hbar_{3}\right) \ldots .\right. \\
& \left.\quad \max \psi_{\check{D}}\left(\hbar_{x-1}, \hbar\right): c, \hbar_{1}, \hbar_{2}, \ldots, \hbar_{x-1}, \hbar \in \check{C}\right\} .
\end{aligned}
$$

Definition 23. A pair $F=(\check{C}, \check{D})$ is said to be a $\mathrm{q}-\mathrm{ROFG}$; then the complete $\mathrm{q}$-ROFG is defined as $\xi_{\check{D} \text { ij }}=\min \left\{\xi_{\check{C} \mathrm{i}}, \psi_{\check{C}_{\mathrm{C}}}\right\}$ and $\psi_{\check{D}_{\mathrm{ij}}}=\max \left\{\xi_{\check{C} \mathrm{i}}, \psi_{\check{C}_{\mathrm{j}}}\right\}$ for every $c_{i}, c_{j} \in \check{C}$.

Definition 24. For a q-ROFG $F=(\check{C}$, $\check{D})$, an edge $(c, \hbar)$ is said to be an SE if $\xi_{\check{D}}(c, \hbar) \geq \xi_{\check{D}}^{\infty}(c, \hbar)$, and $\psi_{\check{D}}(c, \hbar) \geq \psi_{\check{D}}^{\infty}(c, \hbar)$. If there is an SE in $c$, $\hbar \in \check{C}$, we say that $c$ dominates $\hbar$. A node's neighbor is denoted by $N(c)=\{\hbar \in \check{C}:(c, \hbar)$ is a strong edge $\} . \check{S} \subseteq \check{C}$ is assumed to be DS in $F$ if, $\forall \hbar \in \check{C}-\check{S}, c \in \check{S}$ such that $c$ dominates $\hbar$. If no proper subset of a DS occurs, it is called a minimal DS $\check{S}$, and the subset of $\check{S}$ is DS cardinality. The lowest cardinality among all minimal DS is said to be the lower-dominant number (LDN) of $F$ and is represented by $k_{L}(F)$. The upper dominant number (UDN) is defined as the maximum cardinality among all DS, and it is represented by $K_{U}(F)$. If there is no edge connecting the vertices, they are autonomous. A subset $\check{S} \subseteq \check{C}$ is an autonomous set of $F$, if $\xi_{\check{D}}(c, \hbar)<\xi_{\check{D}}^{\infty}(c, \hbar)$, and $\psi_{\check{D}}(c, \hbar)<\psi_{\check{\mathrm{D}}}^{\infty}(c, \hbar)$, where $c, \hbar \in \check{S}$. If the set $\check{S} \bigcup\{c\}$ is not independent for every vertex $\hbar \in \check{C}-\check{S}$, the independent set $\breve{S}$ of $F$ is assumed to be maximally independent. If each vertex $c \in \check{C}-\mathrm{K}$, the set $\check{S} \bigcup\{c\}$ is not independent. For a minimum DS of $F$, if $\check{C}-\mathrm{K}$ contains a DS $K^{-1}$ of $F$, then $K^{-1}$ is said to be the inverse of DS of $F$ concerning $K$. Inverse dominating number $M^{-1}(F)$ of $F$ is the cardinality of a minimum inverse DS of $F$. If a vertex $c \in \check{C}$ has merely one strong neighbor in $F$, it is known as an end-vertex.

\section{Double Domination in q-Rung Orthopair Fuzzy Graph}

In this part, we analyze the DDT of q-ROFGs. The idea of a DDS is described and illustrated with instances. The cardinality of DDS is investigated and the idea of minimal DD and maximal cardinality is studied.

Definition 25. Let $F=(\check{C}, \check{D})$ be a $\mathrm{q}-\mathrm{ROFG}$ and $\mathrm{K} \subseteq \check{C}$, where $\mathrm{K}$ is said to be DDS of $F$ if each vertex in $\mathrm{K}-\check{C}$ has minimum couple of vertices dominant in $\mathrm{K}$. The double domination number (DDN) of $F$ is described as the minimum fuzzy cardinality of all DDS of $F$ and it is represented by $M_{k k}(F)$.

Example 5. Let $F=(\check{C}$, $\check{\mathrm{D}})$ be a $\mathrm{q}-\mathrm{ROFG}$ in Figure 5, and $\mathrm{C}=\left\{c_{1}, c_{2}, c_{3}, c_{4}, c_{5}, c_{6}\right\}$ is a collection of vertices and $\mathrm{D}=$ $\left\{d_{1}, d_{2}, d_{3}, d_{4}, d_{5}, d_{6}\right\}$ is a set of edges.

In Figure 5, we find out the DDN, where $n=3 d_{1}, d_{2}, d_{3}$, and $d_{4}$ are strong edges (SE) and the minimum dominating set $\left\{c_{1}, c_{2}, c_{3}, c_{4}, c_{5}\right\}$ is a DDS of $F$. So $\check{C}-K=\left\{c_{6}\right\}$ and the $\mathrm{DDN}$ is $M_{k k}(F)=3.1$

Theorem 2 shows the presence requirements for double domination and is presented below.

Theorem 2. If the vertex in $\check{C}-K$ has at least two strong neighbors in a q-ROFG F, then DDS occurs in F. 


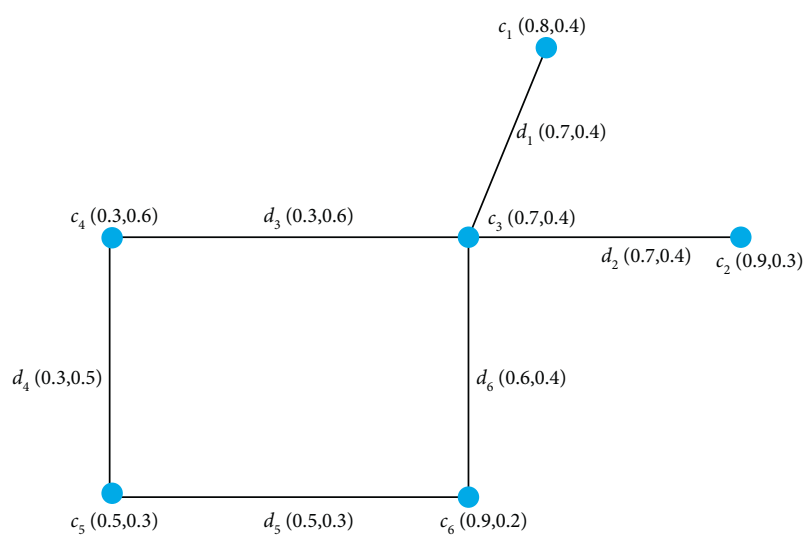

FIgUre 5: Graph with double domination number.

Proof. Let $K$ be a DDS. If a vertex $c \in \check{C}-\mathrm{K}$ includes only one strong neighbor, while further vertices $\check{C}-\mathrm{K}$ have a minimum of two strong neighbors, then for all $c \in \check{C}-\mathrm{K}$, there is a vertex $\hbar \in K$ such that $K$ is DS. This is an inconsistency, and so our assumption is incorrect. Therefore, each vertex in $\check{C}-\mathrm{K}$ should hold a minimum of two strong neighbors.

Example 6. In Figure 6, $F=(\check{C}$, $\check{\mathrm{D}})$ is a q-ROFG, where $\check{\mathrm{C}}=$ $\left\{c_{1}, c_{2}, c_{3}, c_{4}\right\}$ is a combination of vertices and $\mathrm{D}=\left\{\mathrm{d}_{1}, \mathrm{~d}_{2}, \mathrm{~d}_{3}, \mathrm{~d}_{4}, \mathrm{~d}_{5}\right\}$ is a combination of edges.

Here $n=3 d_{1}, d_{2}$, and $d_{5}$ are SEs and $K=\left\{c_{1}, c_{3}, c_{4}\right\}$. So $U-K=\left\{c_{2}\right\}$, and hence $c_{2}$ possesses at least a couple of strong neighbors seen in Figure 6.

Theorem 3 demonstrates the cardinality relation.

Theorem 3. If $F$ is a $q-R O F G$ and $K$ is a DDS in $F$, then $|K| \geq|\check{C}-K|$.

Proof. By DD of $K$, each vertex $\hbar$ in $\check{C}-\mathrm{K}$ requires at least two vertices in $K$ and each $\hbar$ 's neighbor will appear in $\check{D}$. Furthermore, assume that the vertex is strong, and further dominating sets can be produced and neighboring vertices of $\hbar$ will appear in $\check{D}$. Hence, $|K| \geq|\check{C}-\mathrm{K}|$.

Example 7. Suppose that $F=(\check{\mathrm{C}}, \stackrel{\mathrm{D}}{)})$ is a q-ROFG in Figure 7 , and $\check{C}=\left\{c_{1}, c_{2}, c_{3}, c_{4}, c_{5}, c_{6}\right\}$ is a set of vertices and $\grave{D}=\left\{\mathrm{d}_{1}, \mathrm{~d}_{2}, \mathrm{~d}_{3}, \mathrm{~d}_{4}, \mathrm{~d}_{5}\right\}$ is a collection of edges.

In Figure $7, n=4$ and all the edges are SEs and $K=\left\{c_{1}, c_{3}, c_{5}, c_{6}\right\}$. So $\check{C}-K=\left\{c_{2}, c_{4}\right\}$; therefore, $|K|=4$ and $|\check{C}-\mathrm{K}|=2$, and thus $|K| \geq|\mathrm{C}-\mathrm{K}|$.

Theorem 4 examines the required conditions for minimizing double domination.

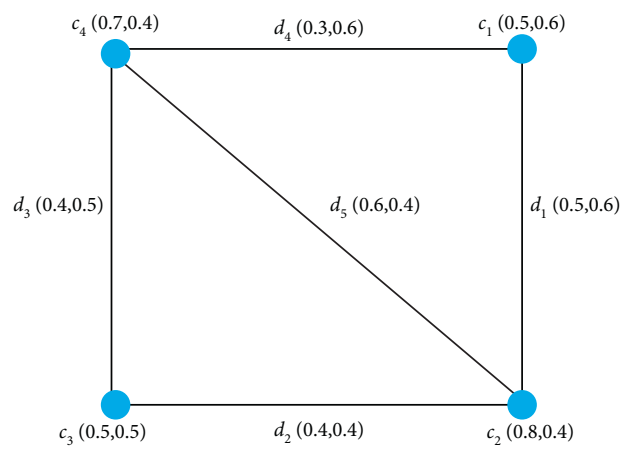

Figure 6: At least two neighbors in $\check{C}-\mathrm{K}$.

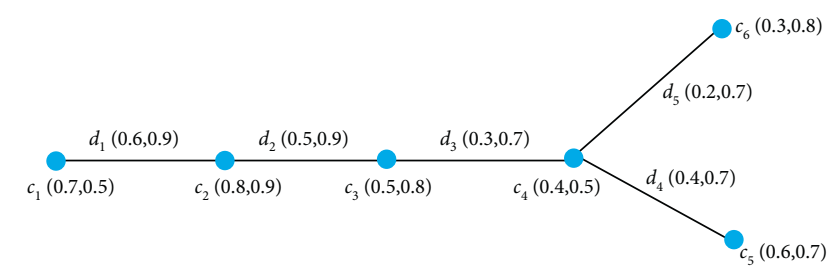

Figure 7: Graph with cardinality.

Theorem 4. The DDS $K$ is a minimal "iff" any two vertices $\{\hbar, \omega\} \in K$; then at least one of its statements is correct.

(i) There exists a vertex $c \in \check{C}-K$, such that $N(\check{C}) \cap K \neq\{\hbar, \omega\}$

(ii) $\check{C}-K$ is isolated

Proof. For minimal DS in q-ROFG $F$, suppose that $\hbar, \omega \in K$ such that $\hbar, \omega$ do not fulfill conditions (1) and (2); suppose that $\mathrm{K}^{\prime}=\mathrm{K}-\{\hbar, \omega\}$ is a DDS fulfilling properties (1) and (2). Hence $\check{C}-K^{\prime}$ is isolated and there is the assumption that $\hbar, \omega \in K$. This supports our statement.

Conversely, for each $\hbar, \omega$ in DDS $K$, at least one of qualities (1) and (2) is true. Suppose on the contrary that $K$ is neot a minimum DDS. Then, $\hbar, \omega \in K$ s.t $K-\{\hbar, \omega\}$ is DDS. As a consequence, $\hbar, \omega$ are adjacent to at least one node in $K-\{\hbar, \omega\}$, implying that $\hbar, \omega$ are weak neighbors to all nodes in $K$. Hence, a node $c \in \check{C}-\mathrm{K}$ s.t $N(c) \cap K \neq\{\hbar, \omega\}$ which is an inconsistency. Thus, $K$ is minimal DDS.

Theorem 5 can be used to establish a relationship between double domination, greatest degree, and least degree correspondingly.

Theorem 5. Let $F=(\check{C}, \check{D})$ be a $q$-ROFG. If $K$ is the minimal DDS, then the following property holds:

$$
\ddot{\mathrm{W}}(K) \leq \delta(F)+2 .
$$


(i) $\ddot{W}(K) \geq \Delta(F)-1$

Here $\ddot{W}(K), \Delta(F)$, and $\delta(F)$ show the weight of $D D S$, greatest degree, and the least degree of $F$ correspondingly.

Proof. Suppose that $K$ is a minimal DDS.

$$
\begin{aligned}
\ddot{W}(K) & =\sum_{1 \leq i \leq n} \min \left(\sum\left[k_{\xi}\left(c_{i}\right)\right], \sum_{1 \leq i \leq n}\left[k_{\psi}\left(c_{i}\right)\right]\right) \\
& \geq\left(\min \left[\sum\left[k_{\xi}\left(c_{i}\right)\right]\right], \min \left[\sum\left[k_{\psi}\left(c_{i}\right)\right]\right]\right) \\
& =\left(\delta_{\xi}(F), \delta_{\psi}(F)\right) \\
& =\delta(F) \\
& \leq \delta(F)+2 \\
\ddot{W}(K) & =\left(\sum \min _{1 \leq i \leq n}\left[k_{\xi}\left(u_{i}\right)\right], \sum \max _{1 \leq i \leq n}\left[k_{\psi}\left(u_{i}\right)\right]\right) \\
& \leq\left(\max \left[\sum\left[k_{\xi}\left(u_{i}\right)\right]\right], \max \left[\sum\left[k_{\psi}\left(u_{i}\right)\right]\right]\right) \\
& =\left(\Delta_{\xi}(F), \Delta_{\psi}(F)\right) \\
& =\Delta(F) \\
& \geq \Delta(F)-1 .
\end{aligned}
$$

Theorem 6. Suppose that $F=(\check{C}, \check{D})$ is a $q-R O F G$ with only end vertex. Then DDS $K$ does not exist.

Proof. For a $\mathrm{q}$-ROFG $F$ containing only end vertices, assume that $K \subseteq \check{C}$. As $F$ is only ended vertex, for every $c \in \check{C}-$ $\mathrm{K} \exists \mathrm{c} \in \mathrm{K}$ such that $K$ is DS.

Furthermore, not any $\check{C}-\mathrm{K}$ vertex is dominated by a minimum of two vertices. As a result, there is not any DDS K.

Example 8. In Figure 8, $F=(\check{C}, \check{\mathrm{D}})$ is a q-ROFG, where $\check{\mathrm{C}}=$ $\left\{c_{1}, c_{2}, c_{3}, c_{4}, c_{5}\right\}$ is a combination of vertices and $\mathrm{D}=\left\{\mathrm{d}_{1}, \mathrm{~d}_{2}, \mathrm{~d}_{3}, \mathrm{~d}_{4}, \mathrm{~d}_{5}, \mathrm{~d}_{6}\right\}$ is a combination of edges.

In Figure $8, n=2, d_{1}$ and $d_{3}$ are the SEs, andthere is not any DDS. So, the other SEs are required to be $d_{2}$ and $d_{4}$.

Theorem 7. For any q-ROFG $F=(\check{C}, \check{D})$, $M_{k k}(F) \geq \mathcal{O}(F) / \Delta_{\psi}(F)+1$, where $\Delta_{\psi}(F)$ is the greatest $\psi$-degree of $F$ and $\mathcal{O}(F)$ represents the order of $q$-ROF.
Proof. Suppose that $K$ is a DDS of q-ROFG $F$ with $|K|=M_{k k}(F)$. While each vertex in $\check{C}-\mathrm{K}$ is nearby to a similar vertex in $K$, we have

$$
\begin{aligned}
|\check{\mathrm{C}}-\mathrm{K}| & \leq \sum_{i=1}^{n} k\left(c_{i}\right) \leq M_{k k}(F) \cdot \Delta_{\xi}(F), \\
\mathcal{O}(F)-M_{k k}(F) & \leq M_{k k}(F) \Delta_{\psi}(F), \\
\mathcal{O}(F) & \leq M_{k k}(F) \Delta_{\psi}(F)+M_{k k}(F), \\
& \leq M_{k k}(F)\left(\Delta_{\psi}(F)+1\right) .
\end{aligned}
$$

This implies that

$$
M_{k k}(F) \geq \frac{\mathcal{O}(F)}{\Delta_{\psi}(F)+1} .
$$

Hence the proof is completed. Theorem 8 illustrates the presence of cut-vertices in DDS.

Theorem 8. If $F$ is a $q$-ROFG with cut-vertex, then DDS $K$ will have a minimum of one cut-vertex.

Proof. For a q-ROFG $F$ with cut-vertex, it is assumed that no cut-vertex occurs in DDS $K$. Consider a cut-vertex $c \in \check{C}-\mathrm{K}$. While cut-vertices are usually the last vertices, as a result, $c$ only has one strong neighbor, which is located in $K$. Furthermore, $K$ is not a DDS, since $c$ in $K$ is not dominated by two or more vertices. This is in opposition to our supposition. Consequently, a cut-vertex $c$ should occur in the DDS $K$. The proof is now complete.

Example 9. Let $F=(\check{C}, \check{D})$ be a q-ROFG in Figure 9, and $\check{C}=\left\{c_{1}, c_{2}, c_{3}, c_{4}\right\}$ is a collection of vertices and $\check{\mathrm{D}}=\left\{\mathrm{d}_{1}, \mathrm{~d}_{2}, \mathrm{~d}_{3}, \mathrm{~d}_{4}, \mathrm{~d}_{5}, \mathrm{~d}_{6}\right\}$ is a collection of edges.

For $n=2$, note that $d_{1}, d_{2}, d_{4}, d_{5}$ are the SEs and $K=\left\{c_{1}, c_{3}, c_{4}\right\}$; after that $\check{C}-K=\left\{c_{2}\right\}$; thus $c_{2}$ is a cut-vertex that can be seen in Figure 9.

Theorem 9. Let $F=(\check{C}, \check{D})$ be a $q-R O F G$, and $M^{-1}(F) \leq M_{k k}(F) \leq|C|$, where $M^{-1}(F)$ is a double dominating number.

Proof. Suppose that $F$ is a q-ROFG. By Theorem 3, inverse domination set $K^{-1} \subseteq \check{C}-\mathrm{K},|K| \geq|\check{C}-\mathrm{K}|$, implying that the DDN is higher than the inverse dominating number; that is, $M^{-1}(F) \leq M_{k k}(F)$, and DDS does not have all the vertices of $F$. This means that at least one of the vertices $\hbar$ must be in $\check{C}-\mathrm{K}$. Thus $F-\{\hbar\}$ provides the double domination number. Obviously $M_{k k}(F) \leq|C ̌|$. Hence proved.

Theorem 10. For $F$ being a $q$-ROFG, DDS $K$ in $F$ is independent but not in $\bar{F}$. 


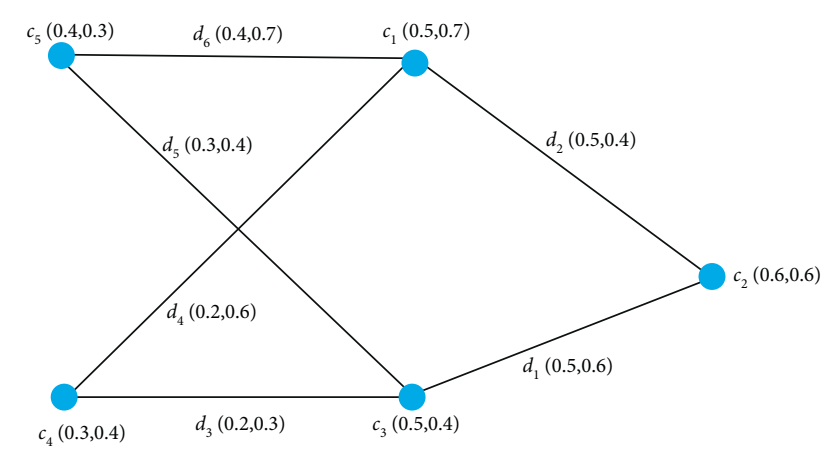

Figure 8: Graph $F$ with only end nodes.

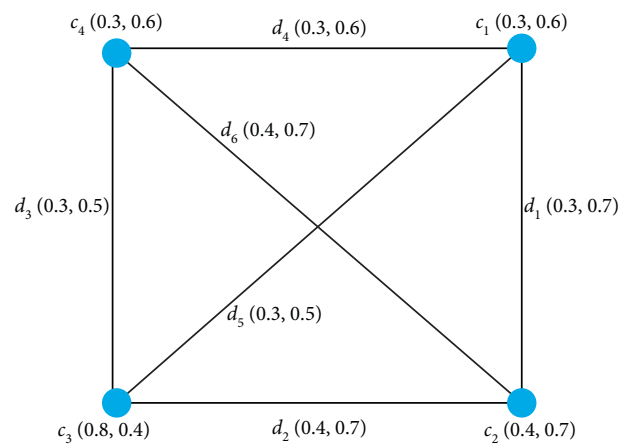

Figure 9: Graph of a DDS with at least one cut-vertex.

Proof. In q-ROFG with an independent DDS, it is assumed that $\bar{F}$ is the complement of $F$, and $\overline{\overline{\check{C}}}=\check{C}$.

$\overline{\xi_{\check{C} i j}}=\min \left(\overline{\xi_{\check{C} \mathrm{i}}}, \overline{\xi_{\check{C} \mathrm{j}}}\right)-\xi_{\text {Čij }}$, and $\overline{\psi_{\text {Dij }}}=\max \left(\overline{\psi_{\text {Di }}}, \overline{\psi_{\text {Dj }}}\right)-\psi_{\text {Dij }}$. Here the only difference is in the values of the edges in $F$. This indicates that neighboring vertices in $\bar{F}$ contain significant neighbors having distinct DDS in $\bar{F}$. Thus, in $F$, the similar DDS $K$ is not independent in $\bar{F}$. The proof is complete now.

\section{Application}

In political races, a lot of the time a politician's target is to achieve as many supporters as feasible in the shortest possible time frame. The idea of a q-rung orthopair DS could be quite useful for this purpose. In general, when a region has a considerable lot of electorates, each elector in that area has registered a node of q-ROFG. By doing so, a large amount of data could be readily managed. Because every node symbolizes an elector, it is possible that two electorates are quite familiar with one another, such as close colleagues or relatives, or that they have just known one another for a short time, or that they are complete strangers. Such relationships between two electorates are indicated by an edge between two vectors. Every edge will display the voting power of two people who are linked to it. If two electorates do not know each other, there is not any edge between them. This is well understood if a political leader contacts a specific elector and secures his support; then, with the assistance of this candidate, he gets accessibility to each of his close friends or colleagues and so there is no need to communicate to each of them separately.

Employing the DT of q-ROFGs, the political leader just wants to convene the member of the DSs of q-ROFGs. Therefore all elements of DS will persuade more members to support the same political leader; thus the majority of electorates can support a certain political leader.

Example 10. Consider the q-ROFG in Figure 10, where we considered a precinct of 7 electorates, showing 7 vertices of q-ROFG, and used edges to discuss their connections. The q-ROFNs are used to represent the values of vertices and edges. $\check{C}=\left\{c_{1}, c_{2}, c_{3}, c_{4}, c_{5}, c_{6}, c_{7}\right\}$ is the total number of vertices and $\left\{d_{1}, d_{2}, d_{3}, d_{4}, d_{5}, d_{6}, d_{7}, d_{8}, d_{9}, d_{10}\right\}$ is the total number of edges. The minimum DS in $F$ is certain by $K=\left\{c_{1}, c_{3}\right\}$; thus, $K-\check{\mathrm{C}}=\left\{c_{2}, \mathrm{c}_{4}, \mathrm{c}_{5}, \mathrm{c}_{6}, \mathrm{c}_{7}\right\}$.

Now the issue can be dealt with by utilizing the q-rung orthopair DS. In order to build q-ROFG, the vertices are designed to denote the electorate in the area. These vertices are associated via edges if the allocated electorates have any relationship. Every relationship between two electorates is given a fuzzy value based on the quality of their interaction. When two people have no relationships, they are considered disconnected.

Utilizing the domination in $\mathrm{q}-\mathrm{ROFG}$, in the graph, there is a minimum DS, as well as the political leader only assembling the participants of that group. Therefore, all DS participants now can demand votes from all non-DS members. Even if the political leader is unable to meet with all of the electorates, his party will receive a majority of the votes. In Figure 10, the political leader only desires to meet the electorate $c_{1}, c_{3}$ for winning an election. Algorithm 1 gives the steps to construct the q-ROFG.

\section{Comparative Study and Advantages}

The dominant idea of q-ROFG will be discussed in this part, which is more flexible than the dominant ideas of FG and IFG. The q-ROFG is an important tool for representing uncertainty and fuzziness. It can be used for enhancing decision-makers capability over orthopairs and their choices. In Example 10, a q-ROFG is discussed wherein the vertices and edges are in the form of q-ROFNs. In this scenario, a q-ROFN is preferable to fuzzy numbers and intuitionistic fuzzy numbers for representing doubtful conditions. Consider the IFG in Figure 11. This graph can be understood as an FG with neutral and a level of nonmembership equivalent to zero. Nonetheless, if we look at the q-ROFG shown in Figures 11 and 12, it cannot be addressed by using ideas of FG and IFG, whereas a q-ROFG may or may not be considered FG or IFG

Furthermore, FG and IFG are unable to deal with the issue raised previously, since these frameworks are bound to certain types of grades. On the other side, if we try and replicate this research in the domain of q-rung orthopair fuzzy environment, we will find that it fails owing to structural restrictions. On the other hand, if we try to do this 
(1) In the q-ROFNs configuration put all the values, including, LM and LNM all the vertices

(2) Using q-ROFGs, describe all of the edges

(3) Draw a contrast of all the edge's values

(4) Construct the power for all edges

(5) Outcome

Algorithm 1: The main steps to construct the q-ROFG.

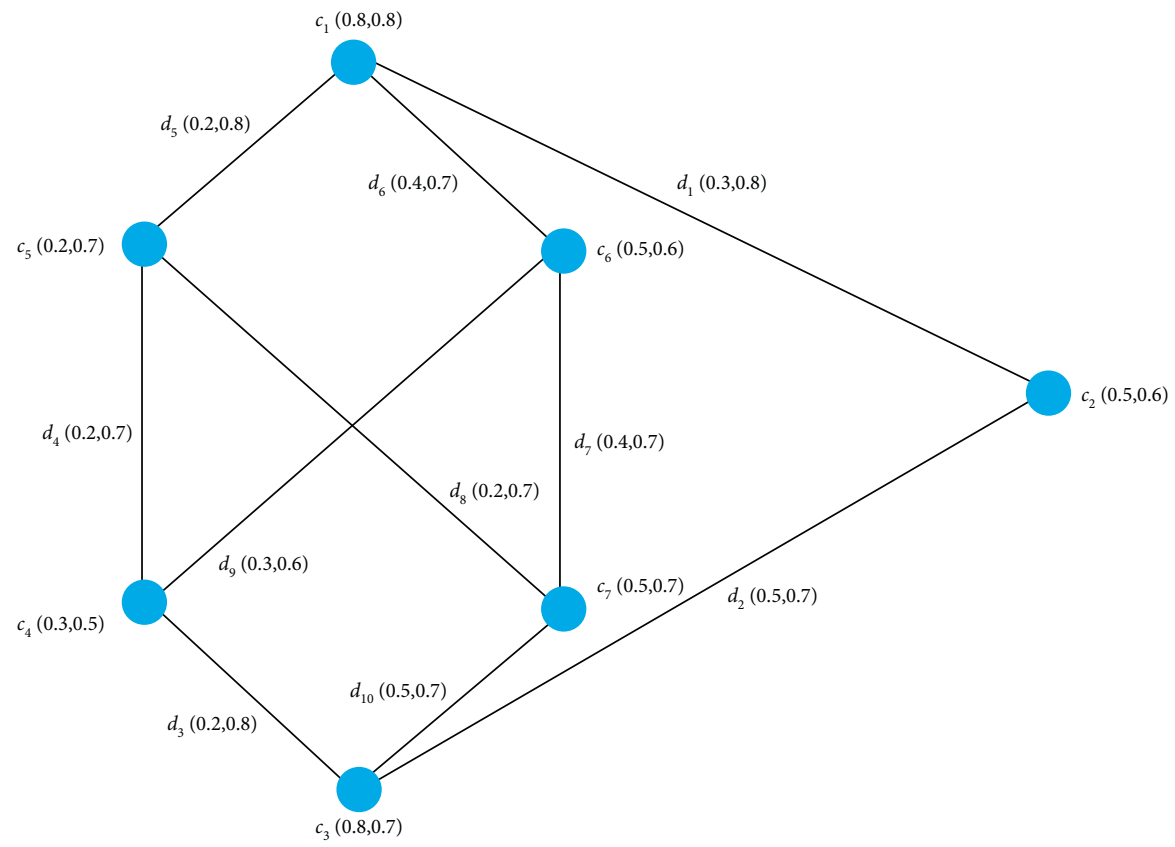

FIgURE 10: The q-rung orthopair fuzzy graphs for analysis.

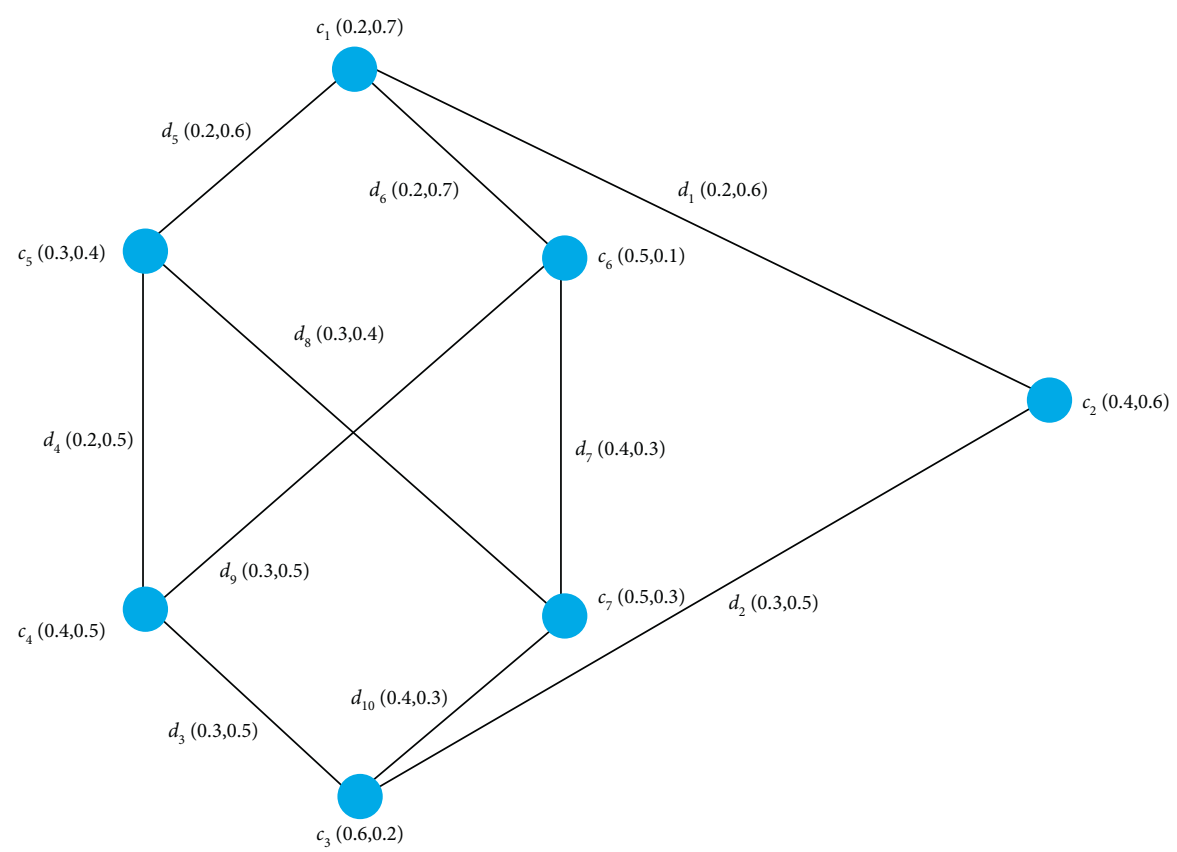

FIGURE 11: IFG for analysis. 


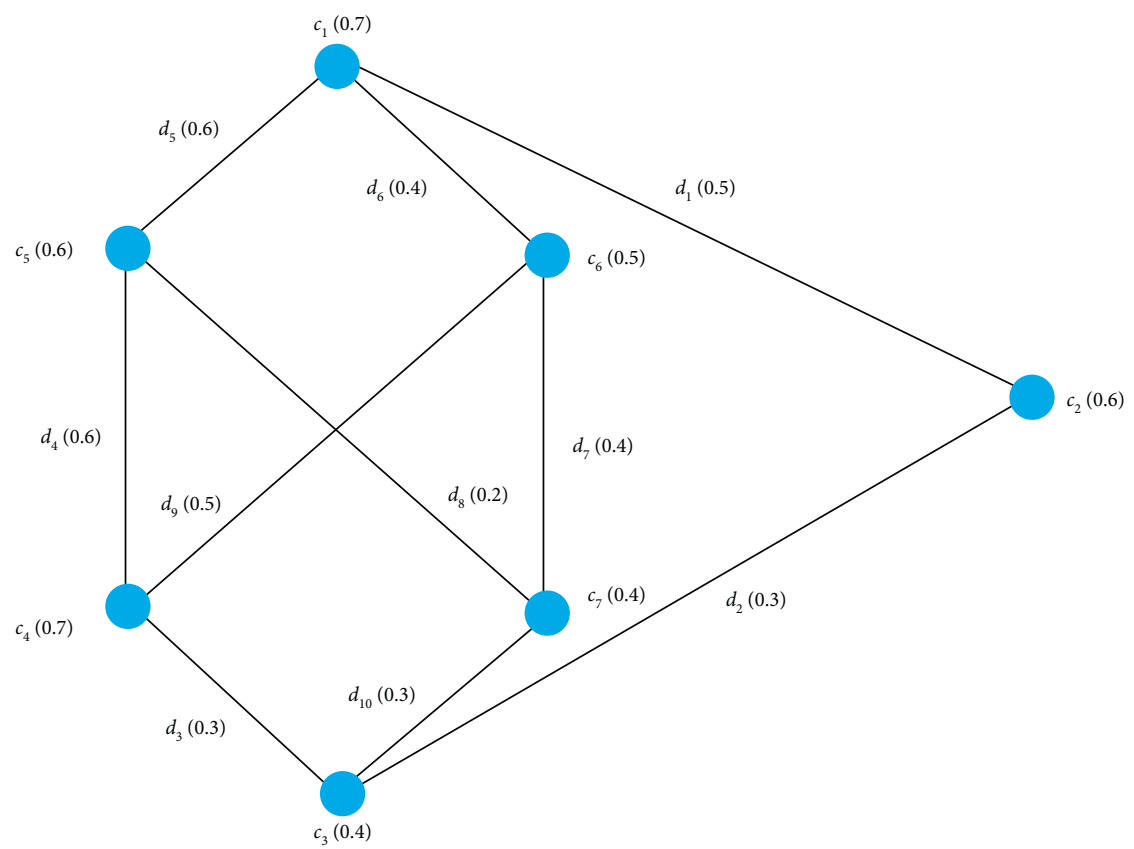

Figure 12: FG for analysis.

TABLE 1: q-rung orthopair fuzzy information when dealing with fuzzy graphs and intuitionistic fuzzy networks.

\begin{tabular}{lccccc}
\hline Object & Levels & Sum of levels & Explanations & Sum of squares of levels & Explanations \\
\hline$c_{1}$ & $(0.8,0.8)$ & 1.6 & False & 1.28 & 0.61 \\
$c_{2}$ & $(0.5,06)$ & 1.1 & False & 1.13 & Talse \\
$c_{3}$ & $(0.8,0.7)$ & 1.5 & False & 0.34 & False \\
$c_{4}$ & $(0.3,0.5)$ & 0.8 & True & 0.53 & True \\
$c_{5}$ & $(0.2,0.7)$ & 0.9 & True & 0.61 & True \\
$c_{6}$ & $(0.5,0.6)$ & 1.1 & False & 0.74 & True \\
$c_{7}$ & $(0.5,0.7)$ & 1.2 & False & 0.73 & True \\
$d_{10}$ & $(0.3,0.8)$ & 1.1 & False & 0.74 & True \\
$d_{2}$ & $(0.5,0.7)$ & 1.2 & False & 0.68 & True \\
$d_{3}$ & $(0.2,0.8)$ & 1 & True & 0.58 & 0.68 \\
$d_{4}$ & $(0.3,0.7)$ & 1 & True & 0.65 & True \\
$d_{5}$ & $(0.2,0.8)$ & 0.8 & True & 0.65 & True \\
$d_{6}$ & $(0.4,0.7)$ & 1.1 & False & 0.85 & True \\
$d_{7}$ & $(0.4,0.7)$ & 1.1 & False & True \\
$d_{8}$ & $(0.2,0.9)$ & 1.1 & True & 0.65 & True \\
$d_{9}$ & $(0.3,0.6)$ & 0.9 & False & 0.74 \\
$d_{10}$ & $(0.5,0.7)$ & 1.2 & & True \\
\hline
\end{tabular}

research in the framework of fuzzy information or intuitionistic fuzzy information, we will most likely fail due to structural restrictions. Table 1 provides a thorough examination of the topic.

\section{Conclusion}

A new concept of q-ROFGs is suggested in this paper. This innovative notion enables us to extend all notions such as FGs and IFGs. Moreover, we conceptualized and modified the concept of double domination theory which elaborates all the currently accessible ideas in graph theory for diverse structures. Basic operations such as cardinality, order, strength, and completeness on dominance, bipartite q-ROFG, and DDS are introduced and demonstrated. This research proposed the ideas of dominant and DDSs, as well as a study of associated terms based on examples. In addition, associated terminologies of q-ROFGs have been defined based on their attributes. The DT is demonstrated in conditions of an election campaign study. A political leader seeks to contact as many electors as possible in a short period. The idea of q-rung orthopair domination could be very useful in this particular skill scenario. The relative examination revealed that the planned framework is 
innovative and aids us in resolving the deficiencies of already available concepts to deal with a situation where other instruments fail to work. We intend to investigate the expansion and fuse of the q-ROFS graph forms in relation to certain application scenes in future study.

\section{Data Availability}

Data sharing does not apply to this article as no data sets were generated or analyzed during the current study.

\section{Conflicts of Interest}

The authors declare that they have no conflicts of interest regarding the publication of the research article.

\section{Acknowledgments}

This research was supported by a Researchers Supporting Project number (RSP-2021/244), King Saud University, Riyadh, Saudi Arabia.

\section{References}

[1] L. A. Zadeh, "Fuzzy sets," Information and Control, vol. 8, no. 3, pp. 338-353, 1965.

[2] K. T. Atanassov, "Intuitionistic fuzzy sets," Fuzzy Sets and Systems, vol. 20, no. 1, pp. 87-96, 1986.

[3] R. R. Yager, "Pythagorean fuzzy subsets," in Proceedings of the IFSA World Congress and NAFIPS Annual Meeting (IFSA/ NAFIPS), June 2013.

[4] R. R. Yager, "Generalized orthopair fuzzy sets," IEEE Transactions on Fuzzy Systems, vol. 25, no. 5, pp. 1222-1230, 2016.

[5] A. Kaufmann, Introduction à la théorie des sous-ensembles flous à l'usage des ingénieurs, Éléments théoriques de base, Masson. 1, France, 1973.

[6] A. Rosenfeld, Fuzzy Graphs, in Fuzzy Sets and Their Applications to Cognitive and Decision Processes, Elsevier, Amsterdam, Netherlands, pp. 77-95, 1975.

[7] K. Sameena and M. S. Sunitha, "Fuzzy graphs in fuzzy neural networks," Proyecciones (Antofagasta), vol. 28, no. 3, pp. 239-252, 2009.

[8] M. Sunitha and S. Mathew, "Fuzzy graph theory: a survey," Annals of Pure and Applied mathematics, vol. 4, no. 1, pp. 92-110, 2013.

[9] R. T. Yeh and S. Y. Bang, Fuzzy Relations, Fuzzy Graphs, and Their Applications to Clustering Analysis, in Fuzzy Sets and Their Applications to Cognitive and Decision Processes, Elsevier, Amsterdam, Netherlands, pp. 125-149, 1975.

[10] R. Parvathi and M. Karunambigai, "Intuitionistic fuzzy graphs," in Computational Intelligence, Theory, and Applications. Springer, pp. 139-150, 2006.

[11] A. A. Talebi, J. Kacprzyk, H. Rashmanlou, and S. H. Sadati, "A new concept of an intuitionistic fuzzy graph with applications," Journal of Multiple-Valued Logic and Soft Computing, vol. 35, 2020.

[12] P. Rangasamy, M. Akram, and S. Thilagavathi, "Intuitionistic fuzzy shortest hyperpath in a network," Information Processing Letters, vol. 113, no. 17, pp. 599-603, 2013.

[13] M. Akram and N. O. Al-Shehrie, "Intuitionistic fuzzy cycles and intuitionistic fuzzy trees," Sci. World J, 11, vol. 201411 pages, Article ID 305836, 2014.

[14] A. Bozhenyuk, S. Belyakov, M. Knyazeva, and I. Rozenberg, "On computing domination set in intuitionistic fuzzy graph,"
International Journal of Computational Intelligence Systems, vol. 14, no. 1, pp. 617-624, 2021.

[15] J. M. Sigarreta, "Total domination on some graph operators," Mathematics, vol. 9, no. 3, p. 241, 2021.

[16] M. G. Karunambigai, S. Sivasankar, and S. Sivasankar, "Different types of domination in intuitionistic fuzzy graph," Annals of Pure and Applied Mathematics, vol. 14, no. 1, pp. 87-101, 2017.

[17] A. Somasundaram and S. Somasundaram, "Domination in fuzzy graphs-I,” Pattern Recognition Letters, vol. 19, no. 9, pp. 787-791, 1998.

[18] R. Parvathi and G. Thamizhendhi, "Domination in intuitionistic fuzzy graphs," Notes on Intuitionistic Fuzzy Sets, vol. 16, no. 2, pp. 39-49, 2010.

[19] O. Manjusha and M. Sunitha, "Strong domination in fuzzy graphs," Fuzzy Information and Engineering, vol. 7, no. 3, pp. 369-377, 2015.

[20] C. Natarajan and S. Ayyasawamy, "On strong (weak) domination in fuzzy graphs. World Academy of Science," Engineering and Technology, vol. 67, pp. 247-249, 2010.

[21] R. A. Borzooei and H. Rashmanlou, "Domination in vague graphs and its applications," Journal of Intelligent and Fuzzy Systems, vol. 29, no. 5, pp. 1933-1940, 2015.

[22] O. Manjusha and M. Sunitha, "Notes on domination in fuzzy graphs," Journal of Intelligent and Fuzzy Systems, vol. 27, no. 6, pp. 3205-3212, 2014.

[23] J. Y. Zhang, Z.-Q. Liu, and S. Zhou, "Dynamic domination in fuzzy causal networks," IEEE Transactions on Fuzzy Systems, vol. 14, no. 1, pp. 42-57, 2006.

[24] M. M. Shubatah, "Domination in product fuzzy graphs," Advances in Computational Mathematics and Its Applications, vol. 1, no. 3, pp. 119-125, 2012.

[25] N. Jan, M. Aslam, K. Ullah, T. Mahmood, and J. Wang, "An approach towards decision making and shortest path problems using the concepts of interval-valued Pythagorean fuzzy information," International Journal of Intelligent Systems, vol. 34, no. 10, pp. 2403-2428, 2019.

[26] V. Pachamuthu and C. Praveenkumar, "Domination in intuitionistic fuzzy graphs of second type," International Journal of Advanced Research Trends in Engineering and Technology, vol. 8, no. 2, pp. 1-4, 2021.

[27] B. Davvaz, N. Jan, T. Mahmood, and K. Ullah, "Intuitionistic fuzzy graphs of n-th type with applications," Journal of Intelligent and Fuzzy Systems, vol. 36, no. 4, pp. 3923-3932, 2019.

[28] A. Habib and A. Farooq, "q-Rung orthopair fuzzy competition graphs with application in the soil ecosystem," Mathematics, vol. 7, p. 91, 2019.

[29] S. Yin, H. Li, and Y. Yang, "Product operations on q-rung orthopair fuzzy graphs," Symmetry Plus, vol. 11, no. 4, p. 588, 2019.

[30] S. Zeng, Y. Hu, and X. Xie, "Q-rung orthopair fuzzy weighted induced logarithmic distance measures and their application in multiple attribute decision making," Engineering Applications of Artificial Intelligence, vol. 100, Article ID 104167, 2021.

[31] B. Wan, R. Lu, and M. Han, "Weighted average LINMAP group decision-making method based on q-rung orthopair triangular fuzzy numbers," Granular Computing, pp. 1-15, 2021.

[32] X. Peng, H. Huang, and Z. Luo, "q-Rung orthopair fuzzy decision-making framework for integrating mobile edge caching scheme preferences," International Journal of Intelligent Systems, vol. 36, no. 5, pp. 2229-2266, 2021.

[33] X. Peng and Z. Luo, "A review of q-rung orthopair fuzzy information: bibliometrics and future directions," Artificial Intelligence Review, vol. 54, no. 4, pp. 1-70, 2021. 